\title{
A rare presentation of fever, sore throat and painful leg nodules
}

A 38-year-old Chinese man presented with a painful rash over bilateral shins for 2 days. He also had a 5-day history of fever, sore throat and myalgia. There were no other localising symptoms of infection. On systems review, he denied having joint pains, alopecia, weight or appetite loss, or any change in bowel habit. Drug history was unremarkable. He had no recent sick contacts or travel history.

Physical examination revealed tender, non-ulcerated purpuric nodules over bilateral legs extending from ankles and shins up to thighs (Fig. 1A). There were no digital ulcers or infarcts; no evidence of livedo reticularis. Notably, multiple discrete punched-out ulcers with an erythematous rim were seen over the hard palate and posterior pharyngeal wall (Fig. 1B). Similar well-demarcated ulcers were present over the base of his penis and inferior scrotal sac (Fig. 1C). There was no evidence of conjunctival injection, perianal ulcers or active synovitis. Periungual erythema and telangiectasia were absent. Abdomen was soft and nontender on palpation. No evidence of clinical pathergy was seen. A $6 \mathrm{~mm}$ punch biopsy was taken from an indurated purpuric shin nodule, which revealed histological features consistent with a medium vessel vasculitis.

What is the most likely diagnosis?
A. Crohn's disease
B. Behçet's disease
C. Orogenital herpes
D. Syphilis
E. Pemphigus vulgaris

The answer is Behçet's disease (BD). Recurrent aphthous stomatitis is the most common and often first presentation of BD. The oral ulcers measure $5-10 \mathrm{~mm}$ in diameter and are well-demarcated with a yellowish pseudomembrane, clear margins and erythematous rim. They are also often clinically indistinguishable from complex aphthosis, and present similarly to genital ulcers. ${ }^{1}$ In males, they are commonly found over the scrotum, foreskin and penis shaft. In females, the most common site is the labia majora. ${ }^{1}$ Common skin manifestations include erythema nodosum (EN)-like rash, pseudofolliculitis and purpuric papules. ${ }^{2}$ While the EN-like rash is usually found on the anterior shin, it can be present elsewhere (e.g. thighs or buttocks). ${ }^{2}$

Arthritis occurs in more than half of BD patients ${ }^{3}$ and can be mono- or poly-articular. It is typically non-erosive, and most commonly affects the knees, though it can also affect the ankles, elbows and wrist. ${ }^{3}$ Other systemic manifestations include gastrointestinal tract ulceration, variable vessel vasculitis and thrombophlebitis. ${ }^{3}$ Neurological manifestations occur late and portend a poor prognosis. ${ }^{2}$

Histological findings of cutaneous lesions vary and commonly include angiocentric and neutrophilic infiltrates with fibrinoid change, leukocytoclasia, and extravasation of red blood cells to a lobular or mixed septal-lobular panniculitis with a mixed inflammatory infiltrate. $^{4}$

The clinical criteria of the International Study Group for Behçet's Disease are widely used to diagnose BD, given its high sensitivity and specificity. ${ }^{5}$ There must be recurrent oral aphthae, and at least 2 of the following:

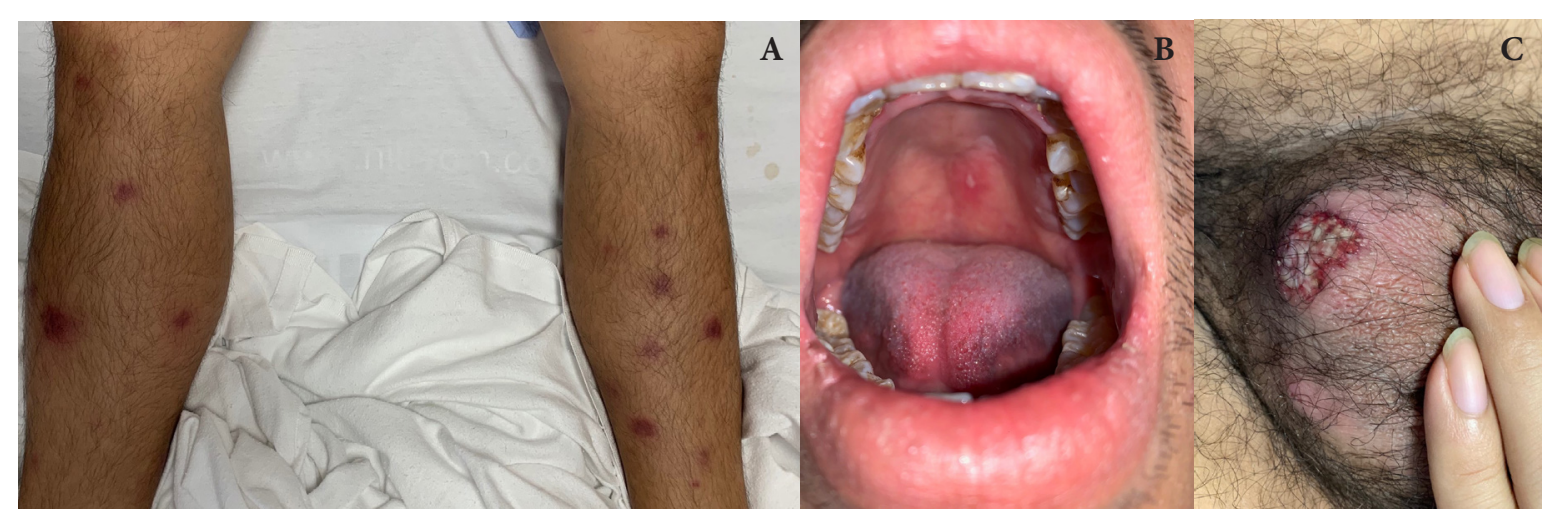

Fig. 1. (A) purpuric shin nodules, (B) oral ulcers, and (C) scrotal ulcer. 
recurrent genital ulceration, ocular involvement (e.g. uveitis), skin lesions (e.g. EN-like lesions, pseudofolliculitis, etc.) and positive pathergy test. ${ }^{5}$

Both EN and recurrent aphthous ulcers can be seen in Crohn's disease. However, perianal ulcers and/or fistulas, rather than scrotal ulcers should be expected. The absence of altered bowel habits also makes this unlikely in our case. Ulcers resulting from grouped vesicles seen in orogenital herpes are often herpetiform with scalloped edges, and the association with EN is uncommon. Primary chancres in syphilis are painless and occur with indurated edges, unlike those shown in Fig. 1. The absence of cutaneous erosions makes pemphigus vulgaris unlikely.

In conclusion, BD is characterised by a constellation of clinical signs and symptoms. Early diagnosis improves overall morbidity.

\section{REFERENCES}

1. Scherrer MAR, Rocha VB, Garcia LC. Behçet's disease: review with emphasis on dermatological aspects. An Bras Dermatol 2017;92:452-64.
2. Davatchi F, Chams-Davatchi C, Shams H, et al. Behcet's disease: epidemiology, clinical manifestations, and diagnosis. Expert Rev Clin Immunol 2017;13:57-65.

3. Sakane T, Takeno M, Suzuki N, et al. Behçet's disease. N Engl J Med 1999;341:1284-91.

4. Kim B, LeBoit PE. Histopathologic features of erythema nodosumlike lesions in Behçet disease: a comparison with erythema nodosum focusing on the role of vasculitis. Am J Dermatopathol 2000;22:379-90.

5. Ferraz MB, Walter SD, Heymann R, et al. Sensitivity and specificity of different diagnostic criteria for Behçet's disease according to the latent class approach. Rheumatol 1995;34:932-5.

Hong Zhe Gabriel Wong ${ }^{1}$, Yee Wei $\underline{\text { Phoon }}^{1}{ }^{M R C P}$

${ }^{1}$ Department of General Medicine, Sengkang General Hospital, Singapore

Correspondence: Hong Zhe Gabriel Wong, Department of General Medicine, Sengkang General Hospital, 110 Sengkang East Way, Singapore 544886.

Email: gabrielwonghongzhe@gmail.com 